\title{
The Effects of Community Size, Control Over Agenda, and Contextual Variables on Zika Virus Preparation of Public Information Officers at Local Public Health Departments
}

\author{
Elizabeth Johnson Avery
}

Department of Communication, University of Tennessee, Knoxville, Tennessee, USA

\begin{abstract}
As Zika emerged as a major global health threat, public information officers (PIOs) at local public health departments across the United States prepared for outbreaks of the virus amid great uncertainty. Using the crisis and risk emergency communication (CERC) model to inform this study, PIOs $(n=226)$ at public health departments were surveyed to assess how community size, perceived control over health agenda, and other considerations such as resources and federal influences affected their satisfaction with Zika preparedness in their departments. These contextual, indirect factors may moderate planning efforts for Zika and other health emergencies and thus should be considered in crisis management and planning models such as CERC.
\end{abstract}

KEYWORDS: Health communication; crisis communication; crisis management; CERC model; Zika virus

Just as Rio de Janeiro prepared to host the 2016 Olympic games, Zika virus was declared a national public health emergency in Brazil. Fear and uncertainty gripped the world as Zika prevalence grew across South America (Umlauf \& Shin, 2016). By summer 2016, the World Health Organization (WHO) had declared Zika an international health emergency. Numerous travel advisories were issued, and pregnant or soon-to-be pregnant women were urged to avoid travel to more than 45 countries, mostly in the Caribbean and Latin America. The first locally transmitted Zika cases in the United States appeared in Florida

CONTACT Elizabeth Johnson Avery•E-mail: ejavery@utk.edu• 476 Communication Building, University of Tennessee, Knoxville, TN 37996, USA 
in July, and the WHO estimated that millions would be affected by the end of 2016 (McNeil, Saint Louis, \& St. Fleur, 2016).

Zika's largest threat was to pregnant women; this became evident as cases of microcephaly, a severe birth defect associated with compromised brain function, increased. Prevalence of Guillain-Barre syndrome also increased in Zika-infected areas (Centers for Disease Control [CDC], 2016b). Even areas without local transmission were at risk of cases resulting from residents traveling to infected areas and from sexual transmission. Zika virus was unfamiliar and mysterious to publics, inducing a higher level of uncertainty (Reynolds \& Seeger, 2005). Villa (2016) noted that, with Zika, "just when the attention of the public is at the top, information about what is going on is usually still missing" (p. 7).

In sum, Zika forced public health officials across the world to manage a global health crisis with potentially devastating effects, especially on unborn children. In late summer 2016, as the first reports of cases in the United States emerged, a survey $(n=226)$ of public information officers (PIOs) at health departments across the United States was conducted to reveal how they were preparing for Zika. Survey data collected during this stage, when the exact nature of the virus and the extent of infection were unrealized for most locales, provide unique insight into planning for health risks emerging as crises. Instead of a retrospective assessment of management, these "real-time" data illuminate the realities of PIOs managing myriad unknowns amid their planning. This study informs health crisis planning by focusing on situational moderators not directly related to the crisis situation itself but affecting PIOs' preparedness to manage Zika outbreaks in their communities, informing a more holistic understanding of crisis readiness and management.

As a comprehensive approach to managing public health emergencies, Reynolds and Seeger's $(2005,2014)$ crisis and emergency risk communication (CERC) model provides a useful framework for this analysis, especially to inform the preparation stage for major health threats. During health crises, the unique operating environments of crisis managers should be carefully considered when making management recommendations. Zika presented complex contingencies at many levels. As Seeger, Reynolds, and Sellnow (2009) noted, greater 
organizational attention to the preparation and coordination phases of crisis management is needed.

The CERC model informs process-based learning from efforts in response to a multifaceted health crisis such as Zika. This study extends current planning considerations in the CERC model by identifying how community size and extent of control over local public health agendas affected PIOs' assessments of their Zika preparedness. Given its complexity and resulting public uncertainty, the case of Zika provides an important contextual framework for this analysis; however, the results also inform preparedness broadly for other health crisis management issues. Furthermore, different barriers to and considerations in PIOs' preparedness efforts are explored to inform a deeper understanding of crisis planning based both on the management process of the crisis itself and moderating factors surrounding it.

\section{Literature Review}

The uncertain trajectory of Zika transmission combined with the threat from travelers returning from infected areas required health officials across the United States to manage an unknown risk emerging as a crisis. With its "process view" of crisis events, Reynolds and Seeger's (2005) CERC model provides a strong framework in which to study the Zika preparedness efforts of a national sample of PIOs at local public health departments who are central to the dissemination and flow of public health communication in their communities. White and Wingenbach (2013) defined PIOs as "communication professionals-often with prior training in journalism-working for nonprofit institutions or government agencies to provide the public and media journalists with information their employers consider crucial to the public good" (p. 123). As important boundary spanners between health experts and the media, PIOs at health departments disseminate often highly technical health information to media and other publics that must be accurately understood and shared (Ankney \& Curtin, 2002). This research supplements and extends the utility of the CERC model by considering external influences on PIOs' planning that could also be incorporated into management recommendations in other stages of the model. 


\section{The CERC Model}

Crisis and risk communication are largely distinct areas of research and practice, but unique health crises require elements of each (Seeger et al., 2009). Crisis communication research focuses on image restoration and response following crisis, from the standpoint of the organization, whereas risk communication entails educating publics on risks and messages designed to change risky behaviors (Seeger et al., 2009). Reynolds and Seeger's (2005) CERC model is a "merged approach that can also be understood as a meta-strategy of crisis preparation and response that informs other strategies and tactics of communication about risks and crises" (Seeger et al., 2009, p. 494). Zika required a dynamic preparedness effort from PIOs as the nature and extent of the threat unfolded and vulnerable publics emerged. Thus preparation efforts, the primary focus of this research, were revised and adapted throughout the process, making Zika a unique context to examine preparedness and to inform planning for other health emergencies as well. The barriers and management considerations explored here are factors external to the crisis itself but very much affecting its management.

\section{The Stages of CERC and the Influence of External Factors Thereon}

The five stages of the CERC model are precrisis, initial event, maintenance, resolution, and evaluation (Reynolds \& Seeger, 2005). The stage of primary interest in this study, the precrisis stage, entails communication targeted to publics and the response community to allow monitoring of risk, public understanding of risk, preparing publics for a negative event, boosting self-efficacy, designing warning messages for a threat, creating alliances with outside partners, developing expert recommendations, and creating messages for later crisis stages.

Additional precrisis considerations investigated in this study are how community size, control over health agenda, and different barriers to crisis planning, such as strained resources, affect PIOs' evaluations of readiness for a major health crisis. These variables are not inherent in the crisis situation but instead affect the ability of the organization to manage it. Ruggiero and Vos (2015) identified resources, competences, and cooperation as critical considerations in crisis planning and crisis 
communication, and they noted that training activities can improve each. Variables such as these may exert a moderating influence over each of the management objectives in the original CERC "working model" (Reynolds \& Seeger, 2005, p. 52). For example, the size of the community and availability of department resources may influence the development of partnerships with external groups and render their management assistance more necessary.

Following the precrisis stage, the initial event stage entails risk messages, warnings, and preparation, as the goal of communication is uncertainty reduction, building self-efficacy, and offering reassurance. In the event stage, the focus narrows onto "reduction of crisis-related uncertainty" (Reynolds \& Seeger, 2005, p. 52), a formidable challenge given PIOs responding to Zika were managing their own considerable uncertainties. As the situation moves into the maintenance stage, message strategy entails ongoing uncertainty reduction and building selfefficacy. Public health communicators must be aware of these stages, present in every crisis or disaster, to anticipate the unique informational needs of various publics, including the general public, the media, and stakeholders. The variables measured in this study supplement CERC by considering the operating environment of the crisis manager and the influence it exerts on management; these variables are most salient to the precrisis and crisis stages of management.

\section{Effects of Community Size on Zika Preparedness}

Resources available to PIOs are one central external management consideration in this study. Major disparities exist between rural and urban populations regarding access to care, staffing, service availability, and financial resources (American Public Health Association, 2016). Resources and information are critical to preparation (Ruggiero \& Vos, 2015; Seeger et al., 2009), yet sustaining the requisite resources and staff to communicate effectively is a particularly challenging aspect of public health event preparedness (Reynolds \& Quinn, 2008). Similar to the challenges presented by pandemic influenza reviewed by Reynolds and Quinn, Zika presented PIOs with a prolonged crisis during which communication needs and response strategies changed based on the stage, presence, transmission, and prevalence of the virus. 
Despite research and policy aiming to reduce health disparities in the United States, they remain a vexing problem (Thompson, Molina, Viswanath, Warnecke, \& Prelip, 2016). More than 50 million underserved people in the United States live in rural and poor urban neighborhoods where health care providers and services are limited (U.S. Department of Health and Human Services [DHHS], 2016). In addition to the health disparities plaguing their populations, PIOs at health departments serving smaller rural areas lag in other areas behind those in larger, better-resourced departments. Finnegan and Viswanath (2008, p. 383) argued that "communication contributes to these circumstances and structural barriers of access and exposure are too frequently ignored or overlooked."

\section{Effects of Public Health Agenda \\ Control on Zika Preparedness}

As a primary source of news for health journalists and through direct promotion, PIOs at health departments contribute greatly to the construction of the local public health agenda. Yet there is evidence of disparities in PIOs' perceived control over their promoted agendas by geography (Avery, Lariscy, \& Sohn, 2009). Health agendas are defined as "systematically structured and articulated sets of goals that guide medical research and training, public policies and spending, and public attention at national, state, and local levels" (Avery et al., 2009, p. 691). Avery and colleagues identified lower levels of perceived departmental control over their health agendas among rural health department PIOs compared to those serving larger populations. PIOs at departments in urban areas reported low levels of state and federal control but high levels of local control over their public health agendas. As evidence of further disparities based on community size, PIOs serving rural health areas reported lower social media adoption rates than those in urban and suburban areas (Avery et al., 2010).

Analyzing the relationship between PIOs' perceived control over their health agendas and their satisfaction with Zika preparedness offers insight into whether and how autonomy in promoting a health agenda influences crisis planning in general. Health agendas can be locally sourced or mandated from a state or federal agency. Almost 30\% 
of states in the United States are operating with a centralized health structure in which a unit of the state health agency provides local health services (CDC, 2016a). This top-down structure may compromise local control in health promotion for the issues identified as most important in a particular community (Avery et al., 2009). In the CERC model, the preparation stage entails monitoring emerging risks and promoting general public understanding of localized risk levels (Reynolds \& Seeger, 2014). Loss of autonomy in promoting the public health agenda and increasing centralization of public health may compromise PIOs' abilities to assess threat levels and develop targeted, localized message strategy. In the context of Zika, similar to other health crises, risk varied widely by geography, illustrating the critical nature of these local assessments.

Quinn (2008) noted that, especially for wide-scale threats, "public health educators engaging in CERC must recognize the risks inherent in disparities to exposure, susceptibility, and treatment and create messages that reflect these realities" (p. 198); local constituents need localized information. Also, as Avery and colleagues (2009) noted, PIOs' perceptions of control in promoting their local health agendas may be an important indicator of the overall well-being of the community public health department, reflected in better initiatives, job satisfaction, and accountability.

The extant body of health and crisis communication literature has pointed out disturbing disparities in the provision of health care and information based on community size. However, no research has directly assessed the impact of community size on preparations for a major health threat. Exploring this relationship may provide a basis for future research generating crisis management directives unique to and considerate of the size of the community the PIO serves. Furthermore, the centralization of public health departments resulting in less locallevel control has been explored for its effects on provision of quality health care and information to publics. However, similar to community size, the direct effects of control over agenda in crisis preparedness have not been identified. To explore the influences of community size and perceived control over the public health agenda on satisfaction with Zika preparedness, the following research question and hypothesis are 
posed, which may extend the utility of CERC to broader moderating considerations in the precrisis stage:

RQ1: What, if any, is the relationship between community size and satisfaction with Zika preparedness?

H1: Extent of control over the local public health agenda will positively predict satisfaction with preparedness efforts.

\section{Considerations in and Barriers to Zika Preparedness}

In addition to examining how community size and control over agenda affect PIOs' satisfaction with their Zika preparedness, this study also considers the various situational barriers and considerations PIOs manage in emergency planning and how they impact planning satisfaction. Examining this relationship during management of the Zika crisis enhances the utility of crisis planning models such as CERC by identifying implications of barriers and unique operating environments on health crisis management. Avery and Graham (2016) argued that recommending a uniform crisis response posture for organizations regardless of external nuances of the situation is unreasonable. The CERC model comprises both process and content dimensions; the process is how those responding to a crisis or emergency evaluate it and create a response (Parmer et al., 2016). It follows that, in those evaluations, crisis managers must take their unique organizational operating environments into account. Zika provided an important contextual opportunity for analysis of that assumption given the uncertainty and complexities it presented to management.

The situational considerations assessed here affect, to some extent, management efforts in every aspect of CERC's precrisis stage. Crisis planning necessitates assessing the vulnerabilities facing the organization (Heath, 2004). Avery and Graham (2016) noted that "even the most tailored recommendations for crisis management are rendered somewhat useless if the organization is unable to implement them due to challenges such as limited budgets or inefficient partnerships, amidst the many other considerations" (p. 21). Their survey of PIOs at local government departments across the United States explored challenges to crisis management unique to the organization, such as 
limited resources. Partnerships with outside agencies were especially important for PIOs in smaller departments with fewer resources to supplement their management efforts during public health threats (Avery \& Graham, 2016). PIOs have reported major failures in their relationships with other health communication practitioners at the local, state, and federal levels (Avery et al., 2009). Yet, underscoring the importance of external partners, Berlin and Carlström (2015) have offered evidence that collaboration exercises with first responders and operational personnel contribute to their learning and the perceived usefulness of the exercises in actual emergency management work. They noted that "by allowing one of the organizations to act in a situation where the professional skills of an absent organization are required, collaborative elements are created" (Berlin \& Carlström, 2015, p. 20). Thus organizations with strained resources will especially realize the value of improved external partnerships in crisis management, an important area to develop in research.

Consideration of resources available to crisis communicators is positioned here as a key area for extending crisis research in health, especially given that PIOs most commonly reported financial barriers to their provision of excellent health care and information in one survey (Avery et al., 2009) and that Ruggiero and Vos (2015) identified resources as critical to understanding crisis planning. Analyzing the importance of different factors affecting crisis planning and how they ultimately influence PIOs' satisfaction with their preparedness offers unique insight to health risk and crisis research. The most influential factors to PIOs' overall satisfaction with Zika preparedness present areas to target in crisis management training for PIOs, and the CERC model could be extended by incorporating recommendations for overcoming challenges. Given that resources and information are vital components of preparation (Seeger et al., 2009) as well as the challenge of maintaining the requisite staff and resources to communicate effectively during crisis (Reynolds \& Quinn, 2008), it is important to use data collected during an emerging major health threat to reveal the experienced impact of resources on preparedness. Thus this study explores factors such as resources required, nature of the crisis, public concern, and advisories from state and federal agencies on Zika virus preparedness by asking the following: 
RQ2: What, if any, underlying factors are there to the following variables affecting crisis management: time it requires, staff/personnel resources, budget resources, severity of crisis, perceived susceptibility of publics, proximity of crisis, public anxiety/fear, state office advisories, federal agency advisories, and citizen concern?

RQ3: How, if at all, does the importance of these factors (RQ2) influence PIOs' levels of satisfaction with their Zika virus preparedness?

\section{Method}

\section{Data Collection}

Upon institutional review board (IRB) approval, an online survey using Qualtrics was created for data collection. First, the survey was pilot tested $(n=15)$ with scholars in the field and members of the target population (i.e., PIOs working at local public health departments, who were excluded from the final survey). Participants were first screened to ensure that they performed communication functions in their public health departments.

An e-mail soliciting participation was sent to 837 e-mail addresses of PIOs obtained by searching local public health department web pages and by calling offices to obtain e-mail addresses of their PIOs. Responses were received from 255 participants for a response rate of $30.5 \%$. Of the 255 who started the e-mail survey, 4 did not give their consent, and 25 participants were omitted, as they did not perform communication functions for their health departments, leaving 226 valid respondents. Of those 226, 18 were partially completed. As compensation for their participation, following IRB-approved procedure, participants were directed to a separate survey not connected to their answers where they could enter their contact information to receive an aggregate report of the data and enter into a drawing to win one of two Apple watches. Finally, the data were cleaned following Morrow and Skolits's (2014) process.

\section{Measures}

Satisfaction with Zika preparedness. Two items measured PIOs' satisfaction with their Zika virus preparedness (Cronbach's $\alpha=.80$ ): 
"My department has allocated sufficient resources toward preparing for a Zika virus outbreak in my community" and "I believe my health department is prepared to safeguard my community if there is a Zika virus outbreak in it." Participants indicated their levels of agreement on a 5-point scale ranging from 1 (strongly disagree) to 5 (strongly agree).

Control over health agendas. Two items, based on measures from Avery and colleagues' (2009) study, were used to measure the extent of control PIOs felt over their local public health agendas (Cronbach's $\alpha=$ .79): "Our local office sets the public health agenda for this community" and "My department has control over the public health issues that are on our local public health agenda." Participants indicated their levels of agreement on a 5-point scale ranging from 1 (strongly disagree) to 5 (strongly agree).

Considerations in crisis planning. Prior research with PIOs (see Avery \& Graham, 2016) used open, in-depth interview questions to generate a list of considerations that affected their crisis management. These items were expanded and used to investigate the extent to which the considerations and, in some cases, barriers to crisis planning influenced satisfaction with Zika preparedness. Participants indicated the level of importance on a 5-point scale ranging from 1 (not at all important) to 5 (very important) of the following considerations in their health crisis planning: time it requires, staff/personnel resources, budget resources, severity of crisis, perceived susceptibility of publics, proximity of crisis, public anxiety/fear, state office advisories, federal agency advisories, and citizen concern.

Community size. PIOs were also asked to classify the locality of the area served by their departments by size in number of inhabitants. These responses were collapsed into six population categories: (a) 9,999 or fewer, (b) 10,000-19,999, (c) 20,000-49,999, (d) 50,000-99,999, (e) 100,000-499,999, and (f) 500,000 or more.

\section{Results}

\section{Sample Profile}

Among respondents, $74 \%(n=147)$ reported their gender as female, $25 \%$ as male $(n=49)$, and $1 \%(n=2)$ as "other." Ages ranged from 22 to 
73 years, with the average age of 48 years $(S D=11)$. There were respondents from 40 U.S. states, representing every major geographic region. Results for the six-category variable of community size were as follows: 9,999 or fewer $(n=12,5.9 \%)$; 10,000-19,999 ( $n=10,4.9 \%)$; 20,00049,999 ( $n=28,13.7 \%)$; 50,000-99,999 ( $n=34,16.7 \%) ; 100,000-499,999$ $(n=66,32.4 \%) ; 500,000$ or more $(n=54,26.5 \%)$. For education, $3 \%$ of PIOs $(n=6)$ had some college but no degree, $2 \%(n=4)$ had an associate's degree, $47.6 \%(n=99)$ had a bachelor's degree, $41.7 \%(n=83)$ had a master's degree, $3 \%(n=6)$ had a doctorate, and $0.5 \%(n=1)$ had a professional degree.

\section{Community Size and Zika Preparedness}

To answer RQ1, analysis of variance (ANOVA) was conducted to reveal group differences in PIOs' satisfaction with their preparedness for Zika based on the independent variable of community size: (a) 9,999 or fewer, (b) 10,000-19,999, (c) 20,000-49,999, (d) 50,000-99,999, (e) 100,000-499,999, or (f) 500,000 or more. Levine's test was not significant, $p=.42$, indicating that the assumption of equality of variances was not rejected. Main effects revealed that preparedness was significantly different among PIOs serving different community sizes, $F(5,202)=8.21, p<.001$. Bonferroni's post hoc tests were conducted to reveal which groups significantly differed. Category $6(M=3.82)$, Category $5(M=3.39)$, and Category $4(M=3.03)$ each had significantly higher means than Category $3(M=2.61)$.

\section{Control Over Local Public Health Agenda and Zika Preparedness}

To test $\mathrm{H1}$, standard regression was conducted to determine whether PIOs' control over their local public health agendas significantly and positively predicted satisfaction with their preparedness for Zika. Tolerance for the IV was assessed and was greater than .1, so the model summary, ANOVA table, and coefficients table were consulted. Regression results indicate that control over local public health agendas significantly predicted satisfaction with preparedness, $R^{2}=.029, F(1,204)=6.13$, $p<.05$. Table 1 presents model coefficients. H1 was supported. 
TABLE 1 Regression Coefficients for Independent Variables

\begin{tabular}{lcccccc}
\hline \multicolumn{1}{c}{ IV } & $\boldsymbol{B}$ & $\boldsymbol{\beta}$ & $\boldsymbol{T}$ & Bivariate $\boldsymbol{P}$ & Partial $\boldsymbol{r}$ & $\boldsymbol{p}$ \\
\hline Population (RQ1)* & 0.19 & 0.31 & 4.6 & 0.31 & 0.31 & 0.00 \\
Control (RQ2)* & 0.20 & 0.17 & 2.48 & 0.17 & 0.17 & 0.01 \\
Threat (RQ3)* & 0.08 & 0.16 & 1.98 & 0.14 & 0.14 & 0.05 \\
Resources (RQ3)* & -0.20 & -0.17 & -2.32 & -0.17 & -0.17 & 0.02 \\
Advisories (RQ3) & 0.08 & -0.08 & -1.02 & -0.08 & -0.07 & 0.31 \\
\hline
\end{tabular}

*Statistically significant independent variable.

\section{Underlying Structure in Considerations for PIOs in Crisis Planning}

Factor analysis was conducted to examine RQ2 and determine what, if any, underlying structure there was to a wide range of barriers to and considerations in PIOs' crisis planning for measures of the following nine variables: time it requires $(M=3.39)$, staff/personnel resources $(M=4.32)$, budget resources $(M=4.07)$, severity of crisis $(M=4.65)$, perceived susceptibility of publics $(M=4.21)$, proximity of crisis $(M=$ 4.32), public anxiety/fear $(M=4.3)$, state office advisories $(M=3.86)$, federal agency advisories $(M=3.72)$, and citizen concern $(M=4.27)$. First, factorability was assessed using the Kaiser-Meyer-Olkin (KMO) index and Bartlett's test of sphericity. The KMO was .735, and Bartlett's test was significant, $p<.001$, indicating the suitability of factor analysis. Initial analyses revealed that citizen concern and severity of crisis (communalities falling below the 6 cutoff, at .428 and .556 , respectively) failed to load on any component or contribute meaningfully to the overall solution and so were excluded from the subsequent analysis (Mertler \& Vannatta, 2005).

Principal components analysis with varimax rotation was conducted, revealing a three-factor solution that explained $72 \%$ of the total variance. The variance accounted for by each of Factors 1, 2, and 3 was $26 \%, 24 \%$, and $22 \%$, respectively. Eigenvalues for each component ranged from 1.2 to 2.8 , satisfying that criterion $(>1.0)$. Component 1 comprised three items, all with positive factor loadings: time it requires (.850), staff/ 
personnel resources (.841), and budget resources (.772). Component 1 was named "Office Resources." Factor 2 comprised three items with positive loadings: public anxiety/fear (.845), proximity of crisis (.808), and susceptibility of publics (.682); it was labeled "Public Threat." The final component comprised state (.894) and federal (.894) advisories. This component was labeled "Agency Advisories."

\section{Factors Affecting Planning Considerations and Zika Preparedness}

To answer RQ3, the three factors were used in a linear regression conducted to explore relationships between crisis planning and satisfaction with Zika preparedness. First, tolerance for each IV was assessed and was greater than .1, so the model summary, ANOVA table, and coefficients table were consulted. Regression results indicate that the overall model significantly predicted satisfaction with Zika preparedness, $R^{2}=.041, F(3,184)=2.63, p<.05$. The regression coefficients of public threat and office resources were significant predictors in the overall model; state and federal advisories were not. See Table 1 for model coefficients.

\section{Discussion}

Zika was a mysterious threat to publics and thus highly uncertainty inducing. A primary goal in the preparation stage of the CERC model is messaging to reduce uncertainty (Reynolds \& Seeger, 2005, 2014), and efforts to inform that goal while considering moderating situational variables that PIOs face are needed. Survey data from PIOs across the United States as they were preparing for Zika in their communities offer a snapshot of real-time risk management and crisis planning, enhancing the validity and value of these results in a way that retrospective reports and assessments of preparedness do not allow. These results extend the utility of the CERC model by considering organizational factors affecting both content and process dimensions of health emergency management using the unique case of Zika. These variables are examined here for their effects on preparedness, but they will likely impact in turn subsequent stages of crisis management. These results 


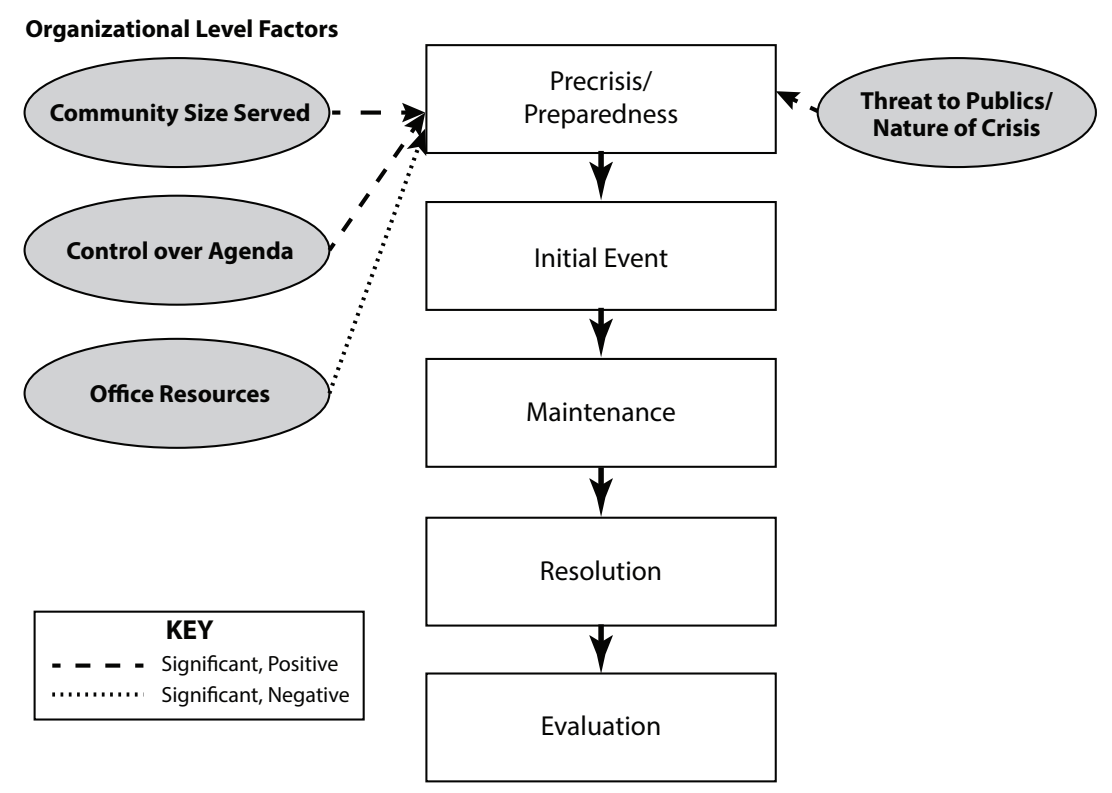

FIGURE 1 CERC model with contextual variables as significant predictors of preparedness.

inform efforts to tailor and adapt best practices to accommodate the various internal and external challenges PIOs face.

Figure 1 presents an expanded CERC model that includes the moderating variables explored in this study external to the crisis situation itself but very much affecting its management. Organizational-level factors such as the size of the community the PIO serves, the PIO's control over the local health agenda, and resources (i.e., time, money, and staff) have been added to the model as important precrisis/ preparedness considerations. These factors are internal yet affect how PIOs prepare for crisis. Externally, characteristics of the threat itself have been added to the model as important factors affecting preparedness. Nature of the crisis variables include public anxiety/fear surrounding the crisis, the proximity of crisis, and susceptibility of the PIOs' publics to the threat. Each of these novel considerations is discussed in turn.

Troublesome disparities in PIOs' satisfaction with their planning were present in both situational and departmental factors. Regarding community size and Zika planning, practitioners serving the largest 
areas (500,000 or more) reported the highest overall satisfaction with their Zika preparedness. PIOs serving smaller community sizes with populations between 50,000 and 99,999 reported the lowest level of satisfaction, and that level was significantly lower than it was for the three largest size categories. This finding merits further examination, as the practitioners serving mid-sized towns fared the worst, and all three of the smallest categories had lower mean satisfaction than the three largest categories. Perhaps initiatives to assist rural areas, such as the DHHS's (2001) Rural Task Force, have enabled the smallest-sized towns to fare slightly better than their more "overlooked" medium-sized town counterparts in crisis management. Overall, there is much room for improvement for PIOs serving both middle- and smaller-sized communities through enhanced CERC-based training efforts and assistance sensitive to their unique needs and operating environments. Thompson and colleagues (2016) noted that "if health disparities are to be reduced or eliminated, the playing field must be leveled" (p. 1427). These results indicate the need for heightened resources and assistance with preparedness in these communities.

Although the reasons for these differences are beyond the scope of this study, it offers disturbing evidence of additional disparities between rural and urban populations in crisis preparedness beyond those previously identified regarding access to care, staffing, service availability, and financial resources (American Public Health Association [APHA], 2016). Services and resources are generally more abundant for departments serving larger areas (APHA, 2016), and, in this case, even perceived preparedness for a major health threat lagged in smaller areas. Given the importance of adequate resources in preparedness (Ruggiero \& Vos, 2015; Seeger et al., 2009), it is not surprising that practitioners in small, often understaffed and underfunded departments lag behind their counterparts at larger departments in planning. PIOs with smaller staffs have less assistance with both health education and crisis management functions, likely resulting in decreased available time to allocate to preparedness. The presence documented here of a possibly overlooked "middle" and the implications of these gaps on public safety demand more scrutiny to identify causation. CERC and other management models can be extended with targeted 
recommendations for managing resource deficits in crisis planning.

Furthermore, PIOs' perceived control over their local public health agendas significantly and positively influenced their assessments of Zika planning: Those who felt more control over promoting the health issues they deemed important in their communities were also more satisfied with their preparedness. It is not surprising that those with more perceived autonomy in their routine health interventions would also be more satisfied with their planning for a specific health threat in their communities. However, this finding is troublesome given that 14 states are currently operating with a centralized health structure (CDC, 2016a). In centralized states, local services are provided by the state agency instead of by autonomous, independent health departments primarily working at the local level with state coordination. The provision of localized risk assessments to communities-especially during wide-scale threats like Zika-is central to CERC-based public health education (Quinn, 2008). Centralization compromises locallevel control, which likely compromises PIOs' abilities to promote the issues they perceive as most pressing in their communities, those both routine and crisis in nature. During the Zika crisis, the extent of threat to publics varied extensively by location in the United States, even within states (such as Florida, where outbreaks were only in the southern portions). The case of Zika thus illustrates the importance of specifically tailored, localized information.

The effects of agenda control on risk and crisis management have not been adequately explored. Deficits may be due to structural and bureaucratic mechanisms or more to local situational considerations. Perhaps strained resources alone compromise control if PIOs do not have the time, staff, or money to promote the agendas they desire. Especially when considered along with the disparities revealed here regarding community size and preparedness satisfaction, the fact that Avery and colleagues (2009) offered evidence of less perceived control among PIOs in rural than urban public health departments is troublesome. This barrier could widen the gap between services availed by smaller, rural departments. Furthermore, CERC-based public health education entails providing localized risk assessments to communities, especially during wide-scale threats like Zika (Quinn, 2008). It follows that PIOs 
with compromised control over promotion of their local public health agendas are also less satisfied with their levels of preparedness overall. If control is also an important indicator of overall health promotion, job satisfaction, and departmental accountability for PIOs (Avery et al., 2009), these relationships demand more causal probing.

Based on qualitative data from experts' evaluations of terrorism crisis management, Ruggiero and Vos (2015) identified resources as a critical area of consideration before and during the crisis. The quantitative data collected in this study support and extend that important contention and position it within the CERC model as a pressing area of development. Office resources, threat levels to publics, and agency advisories emerged as three components underlying PIOs' considerations in crisis planning. Threat to publics and resources required to manage a health crisis significantly predicted satisfaction with Zika preparedness in the regression model. Resources required negatively predicted preparedness satisfaction, while threat positively predicted satisfaction with preparedness. PIOs were more satisfied with their Zika preparedness as perceived importance of anxiety/fear levels of publics, the proximity of the crisis, and the susceptibility of publics in crisis planning increased.

This finding was positive in that PIOs were increasingly satisfied with their planning efforts with higher perceived threat levels; they were responding to the challenge in a satisfying way. More bleak is the significant negative relationship between resources (including time, money, and staff) and Zika preparedness: As importance of those considerations in crisis planning increased, satisfaction decreased. PIOs for whom resources were important, likely compromising their crisis planning efforts, were not as satisfied with their Zika preparedness. Again, research-based direction for accommodating resource barriers in crisis management is needed. CERC provides a very useful health crisis management model that can be expanded to include accommodating directions for resource and other structural obstacles PIOs face to enhance its utility. Although it is beyond the scope of this study to make specific recommendations in the CERC model on accommodating strained resources, precrisis steps within CERC, such as forming alliances and cooperating with other organizations, may be expanded 
to assist underresourced PIOs. Partnerships with external organizations may yield critical assistance for PIOs at small departments managing crisis. Steps for establishing connections to supplement strained crisis management areas within the department and having strong partnerships in place precrisis may be useful extensions of CERC.

\section{Limitations and Future Research}

Several of the limitations of this research are inherent to any survey research. These results do not enable causal attributions for why some of these results are the case. Also, the overall explained variance for several of the regression equations was quite low. For the incredibly complex processes involved in the dependent variables, however, this is to be expected. The goal was less to develop overall predictive models and more to understand if and how certain considerations factored into Zika preparation to provide direction for future research. Another limitation is that this study was based on self-reported data.

Overall, this research investigates PIOs' planning for a major public health crisis, Zika virus, that was shrouded in unknowns by collecting data in real-time as PIOs at local health departments across the United States prepared for crisis. Future research needs to employ a co-orientational approach to identify discrepancies between PIOs' and their publics' understandings and evaluations of crisis preparation and response. The disparity in planning satisfaction demonstrated by PIOs serving smaller areas reveals a pressing area for intervention. Given that extent of perceived control over local public health agendas significantly influenced satisfaction with Zika planning and that almost one-third of states are going to a more centralized model (CDC, 2016a), the effects of centralization and how it compromises control and thus health crisis preparedness demand more scholarly attention.

\section{Conclusion}

This research adopts and extends the CERC model with process and content dimension considerations that affect every stage of risk and crisis management for PIOs at local health departments through insight provided by their preparation for a global health threat. Overall, 
PIOs serving larger communities were more satisfied with Zika preparedness, and their satisfaction with preparedness increased as their perceived control over their health agendas increased. The threat to publics and office resources were significant predictors of satisfaction with preparedness. Taken together, these results present unique and important considerations for understanding preparedness for major health crises and the opportunity to use research-based recommendations to improve PIOs' crisis preparation, particularly among PIOs at smaller departments and with more strained resources.

Elizabeth Johnson Avery, $\mathrm{PhD}$, is a professor of public relations and was codirector of the Risk, Health, and Crisis Communication Organized Research Unit at the University of Tennessee from 2009 to 2013. Avery is currently working with funding from the U.S. Department of Health and Human Services to address the opioid crisis in rural Appalachia. Prior to joining the faculty at the University of Tennessee, Avery completed a postdoctoral assistantship on a \$3.5 million CDC grant, served as campaign manager for a mayoral candidate, and was the environmental education coordinator at the Athens-Clarke County Recycling Division for several years.

\section{ORCID}

Elizabeth Johnson Avery (D) https://orcid.org/oooo-0002-2565-3521

\section{References}

American Public Health Association. (2016). Rural health goals: Guaranteeing a future. Retrieved from http://www.apha.org/policies-and-advocacy /public-health-policy-statements/policy-database/2014/07/30/10/36/rural -health-goals-guaranteeing-a-future

Ankney, R. N., \& Curtin, P. A. (2002). Delineating (and delimiting) the boundary spanning role of the medical public information officer. Public Relations Review, 28, 229-241. https://doi.org/10.1016/So363-8111(02)o0129-7 Avery, E. J., \& Graham, M. (2016). Developing an integrated crisis con- 
text approach for crisis management. Public Relations Journal, 10, 1-21. Avery, E. J., Lariscy, R. W., \& Sohn, Y. (2009). Public information officers' and journalists' perceived barriers to providing quality health information. Health Communication, 24, 327-336. https://doi.org/10.1080/1041023090 2889365

Avery, E., Lariscy, R., Amador, E., Ickowitz, T., Primm, C., \& Taylor, A. (2010). Diffusion of social media among public relations practitioners in health departments across various community population sizes. Journal of Public Relations Research, 22, 336-358. https://doi.org/10.1080/106272610036 14427

Berlin, J. M., \& Carlström, E. D. (2015). Collaboration exercises: What do they contribute? Journal of Contingencies and Crisis Management, 23, 11-23. https://doi.org/10.1111/1468-5973.12064

Centers for Disease Control and Prevention. (2016a). State health departments governance details. Retrieved from https://www.cdc.gov/stltpublichealth /docs/sitesgovernance/public-health-governance-details.pdf

Centers for Disease Control and Prevention. (2016b). Top 10 Zika response planning tips: Brief information for state, tribal, local, and territorial health officials. Retrieved from http://www.cdc.gov/zika/public-health-partners /tips.html

Finnegan, J. R., \& Viswanath, K. (2008). Communication theory and health behavior change. In K. Glanz, F. Lewis, \& B. Rimer (Eds.), Health behavior and health education: Theory, research and practice (pp. 361-388). San Francisco, CA: Jossey-Bass.

Heath, R. L. (2004). Crisis preparation: Planning for the inevitable. In R. Heath \& D. Miller (Eds.), Responding to crisis: A rhetorical approach to crisis communication (pp. 1-17). Hillsdale, NJ.: Erlbaum.

McNeil, D., Saint Louis, C., \& St. Fleur, N. (2016, July 29). Short answers to hard questions about Zika virus. New York Times. Retrieved from http:// www.nytimes.com/interactive/2016/health/what-is-zika-virus.html

Mertler, C. A., \& Vannatta, R. A. (2005). Advanced and multivariate statistical methods. Glendale, CA: Pyrczak.

Morrow, J. A., \& Skolits, G. (2014, November). The twelve steps of data cleaning: Strategies for dealing with dirty data. Workshop presented at the annual meeting of the American Evaluation Association, Denver, CO.

Parmer, J., Baur, C., Eroglu, D., Lubell, K., Prue, C., Reynolds, B., \& Weaver, J. 
(2016). Crisis and emergency risk messaging in mass media news stories: Is the public getting the information they need to protect their health? Health Communication, 31, 1215-1222. https://doi.org/10.1080/10410236.2015 .1049728

Quinn, S. C. (2008). Crisis and emergency risk communication in a pandemic: A model for building capacity and resilience of minority communities. Health Promotion Practice, 9(Suppl. 4), 18s-25s. https://doi .org/10.1177/1524839908324022

Reynolds, B., \& Quinn, S. C. (2008). Effective communication during an influenza pandemic: The value of using a crisis and emergency risk communication framework. Health Promotion Practice, 9(Suppl. 4), 135-17s. https://doi.org/10.1177/1524839908325267

Reynolds, B., \& Seeger, M. W. (2005). Crisis and emergency risk communication as an integrative model. Journal of Health Communication, 10, 43-55. https://doi.org/10.1080/10810730590904571

Reynolds, B., \& Seeger, M. W. (2014). Crisis and emergency risk communication (2014 ed.). Retrieved from https://stacks.cdc.gov/view/cdc/25531.

Ruggiero, A., \& Vos, M. (2015). Communication challenges in CBRN terrorism crises: Expert perceptions. Journal of Contingencies and Crisis Management, 23, 138-148. https://doi.org/10.1111/1468-5973.12065

Seeger, M. W., Reynolds, B., \& Sellnow, T. L. (2009). Crisis and emergency risk communication in health contexts: Applying the CDC model to pandemic influenza. In R. L. Heath \& H. D. O’Hair (Eds.), Handbook of risk and crisis communication (pp. 493-506). New York, NY: Routledge.

Thompson, B., Molina, Y., Viswanath, K., Warnecke, R., \& Prelip, M. L. (2016). Strategies to empower communities to reduce health disparities. Health Affairs, 35, 1424-1428. https://doi.org/10.1377/hlthaff.2015.1364

Umlauf, T., \& Shin, Y. (2016). How big a threat will Zika be at the Olympics. Wall Street Journal. Retrieved from http://graphics.wsj.com/threat-of -zika-at-the-rio-olympics/

U.S. Department of Health and Human Services. (2001). HHS issues report on community health in rural, urban areas. Retrieved from http://www.hhs.gov /news/press/2001pres/20010910.html

U.S. Department of Health and Human Services. (2016). Health Resources and Services Administration. Retrieved from http://www.hrsa.gov/about /budget/budgetjustification2016.pdf 
Villa, R. (2016). Zika, or the burden of uncertainty. La Clinica Terapeutica, 167, 7-9. https://doi.org/10.7417/CT.2016.1907

White, J. M., \& Wingenbach, G. (2013). Potential barriers to mass media coverage of health issues: Differences between public information officers and journalists regarding beliefs central to professional behaviors. Journal of Public Relations Research, 25, 123-140. https://doi.org/10.1080/1062726X .2013 .758582 